\title{
Photoactive channels obey stoplights
}

Kinetically bi-stable channelrhodopsin-2 variants enable sensitive, step-like and reversible photoexcitability in neurons.

Light-based tools have changed the landscape in neuroscience research by enabling simultaneous noninvasive and finely localized control of neuronal stimulation. Lightresponsive ion channels called opsins are fundamental to this experimental revolution; neurons that express opsins can be selectively activated, which allows scientists to study how neurons communicate with each other and to map neuronal networks. Karl Deisseroth and his colleagues at Stanford University in collaboration with Peter Hegemann's group at Humboldt University now report new variants of channelrhodopsin-2 (ChR2), a light-activated cation channel from green algae, that enable precise switching between stable active and inactive states.

As Deisseroth explains, these endeavors in molecular engineering were dually inspired: "One interest was in the biophysical mechanism of how the channel works and which residues controlled properties like gating, but the most important new aspect was the practical utility of being able to switch the channels into and out of stable open states with only brief pulses of light."

To engineer channels with such favorable kinetics, the researchers took advantage of structural knowledge of the related bacteriorhodopsin ion pump and existing physical and chemical information about the ChR2 photocycle to target the cysteine at position 128 (Cys128). According to Deisseroth, structural predictions placed Cys128 near the chromophore, all-trans retinal, so it was expected to have some role in governing chromophore behavior, but it was remarkable that the isolated Cys128 substitution effectively changed the kinetics of the channel. The researchers engineered three distinct ChR2 Cys128 mutants; ChR2-C128T, ChR2C128A and ChR-C128S. Although each of the mutant channels had some unique properties, they all exhibited an extended conducting state lifetime, which enabled prolonged and reversible membrane depolarization governed by short light pulses.

Deisseroth expects ongoing efforts to improve key features of these channels. "One disadvantage is that some of the mutants have reduced current compared to wild type, so multiple mutations may help to bring those current levels back up to wild-type levels," he says. Projects designed to improve membrane targeting and to apply a composite of opsins, including the red light-responsive channelrhodopsin from Volvox carteri, are also in the works in his laboratory.

Although other tools are available for localized neuronal stimulation, Deisseroth expects these new channels to provide a powerful alternative. "Chemical methods have shown really beautiful data, but in large mammalian brains, it's difficult to use chemical cofactors and ultraviolet light." Additionally, these specialized channels can be used to sensitize target cells in a way that wild-type channels could not, so studies can access bona fide neuronal responses rather than simply adding artificial stimulation. Repeated activation and inactivation of the engineered channels can increase excitability in a target cell population, which allows native underlying codes to come through more effectively. The ability to stably turn the channels on and off with short pulses of light and the overall increase in light sensitivity "are together fortunately synergistic," Deisseroth adds.

These two features will improve and make possible new applications for ChR2 requiring chronic stimulation. Controlling neuronal activation with low-intensity short light pulses will reduce the amount of energy deposited into a tissue, thereby diminishing damage, and will save on battery life for devices that provide chronic deep brain stimulation (for example, treatment of Parkinson's disease or depression). Deisseroth adds, "Any kind of long-term application, which might include developmental or homeostatic changes as well as clinical or preclinical applications, will benefit greatly from this."

Although Deisseroth is clearly enthusiastic about these new tools, he remains focused on overcoming the remaining technical obstacles that limit neuroscientists, "This is a promising step, but we have a lot more to do."

\section{Amy Donner}

\section{RESEARCH PAPERS}

Berndt, A. et al. Bi-stable neural state switches. Nat. Neurosci. advance online publication 8 December 2008. 